Pacific Journal of Mathematics

COMMUTANTS OF MULTIPLIERS AND TRANSLATION
OPERATORS 


\title{
COMMUTANTS OF MULTIPLIERS AND TRANSLATION OPERATORS
}

\author{
MARTIN BARTELT
}

\begin{abstract}
This note discusses a method for the determination of the commutant of a set of translation operators on the ring of bounded functions in $C(X), X$ locally compact.
\end{abstract}

1. Introduction. If $X$ is a locally compact group, then $X$ can be represented and studied as a class of operators on $C_{b}(X)$. Indeed, if $x \in X$ and $f \in C_{b}(X)$, then one defines an operator $U_{x}$ on $C_{b}(X)$ by $U_{\mathrm{r}} f(y)=f(y+x), \forall f \in C_{b}(X)$. The commutant of $\left\{U_{x}: x \in X\right\}$ was studied in [3] and [4].

The general method of $\$ 3$ for the determination of the commutant of a set of translation operators is applied to the study of the commutant of a class of operators on bounded analytic functions. In $\$ 4$ some directly obtainable results are given on commutants of multiplier operators on bounded analytic functions.

2. Definitions. Let $X$ denote a locally compact Hausdorff space. Let $C(X)$ denote the algebra of continuous complex valued functions on $X$ and let $C_{b}(X)$ denote the subalgebra of $C(X)$ consisting of bounded continuous functions. One can study $C_{b}(X)$ in the topologies $\kappa, \beta$, or $\sigma$; respectively, uniform convergence on compact subsets of $X$, the strict topology or uniform convergence on $X$. The topology $\sigma$ is a norm topology with $\|f\|=\sup _{x \in X}|f(x)|$. The strict topology was introduced in [6] and its properties may be found in [5], [6], [8] and [9]. Particularly relevant here are that the $\sigma$ and $\beta$ bounded subsets of $C_{b}(X)$ coincide; on $\beta$ bounded subsets of $C_{b}(X)$, the strict topology coincides with $\kappa$; and a sequence $\left\{f_{n}\right\}$ converges $\beta$ to zero if and only if it is $\sigma$ bounded and $\kappa$ convergent [6].

Denote by $(C(X)$, c.o. $)$ the space $C(X)$ endowed with the compact open topology or equivalently with the topology of uniform convergence on compact subsets. The space $(C(X \rightarrow X)$, c.o. $)$ is similarly defined.

Let $B$ denote the bounded analytic functions on the open unit disc $D=\{z:|z|<1\}$ in the complex plane. Since $B$ is a $\kappa$ closed subspace of $C_{b}(D)$, one may consider $B$ endowed with any one of the topologies $k, \beta$ or $\sigma$, denoted respectively by $(B, \kappa),(B, \beta)$ and $(B, \sigma)$. The algebra $[\beta: \beta]$ of all continuous linear operators from $(B, \beta)$ into $(B, \beta)$ has been studied in [1] and [2], and it is closely related to an algebra of operators studied in [4]. In particular $[\beta: \beta]$ is a norm closed subalgebra of $[\sigma: \sigma]$ 
the algebra of all continuous linear operators from $(B, \sigma)$ into $(B, \sigma)$ and $[\beta: \beta]$ seems more tractable than the larger algebra $[\sigma: \sigma]$.

We will be concerned with translation operators and the commutant in various spaces of operators of a given set of operators.

Definition. For $\phi$ a continuous map of $X$ into $X$, the translation operator $U_{\phi}$ is defined on $C_{b}(X)$ by

$$
U_{\phi} f(x)=f(\phi(x)) .
$$

Definition. Let $G$ be a set of operators in $[\beta: \beta]$. Then the commutant of $G$ in $[\beta: \beta]$ is

$$
\operatorname{Comm}(G)=\{V \in[\beta: \beta]: T V=V T, \forall T \in G\} .
$$

3. Commutants of operators on $C_{b}(X)$ or on $B$. The first result in this section is a general method for determining the commutant of a set of translation operators on $C_{b}(X)$. We associate with each operator in the commutant a linear functional on $C_{b}(X)$.

Now assume that to each $x \in X$ is associated one and only one continuous map $\phi_{x}$ from $X$ into $X$. In the special case when $X$ is a group, then $\phi_{x}$ might be defined by $\phi_{x}(a)=a+x$ and $X$ is then represented as a group of operators on $C_{b}(X)$. In general we define the operator $U_{\Phi(x)}$ on $C_{b}(X)$ by

$$
U_{\Phi(x)} f(y)=f\left(\phi_{x}(y)\right) \text { where } \quad \Phi(x)=\phi_{x} .
$$

The topology $\sigma$ is not appropriate for our purposes because the map $x \rightarrow U_{x} f$ will not in general be continuous (see [5]). This difficulty is overcome by using the strict topology on $C_{b}(X)$ (see Lemma 2). The following three lemmas follow from the definitions and known properties of the compact open and strict topologies and also from the characterization of continuity via nets.

Lemma 1. Let $S \subseteq X . \quad$ The map $\Phi: S \rightarrow(C(X \rightarrow X)$, c.o. $)$ is continuous if and only if the map $\psi$ is continuous where

$$
\psi: S x X \rightarrow X, \quad \text { with } \quad \psi(x, y)=\phi_{x}(y) .
$$

Lemma 2. Assume that the map $\Phi: X \rightarrow(C(X \rightarrow X)$, c.o. $)$ is continuous. Then the map $\psi: X \rightarrow\left(C_{b}(X), \beta\right)$ is continuous where $f$ is fixed in $C_{b}(X)$ and $\psi(X)=U_{\Phi(x)} f$.

Lemma 3. Let $F=\left\{f_{\alpha}: \alpha \in A\right\}$ where $f_{\alpha} \in C(X \rightarrow X)$. Assume that 
$F$ is compact in $(C(X \rightarrow X)$, c.o. $)$ and let $K$ be a compact set in $X$. Then $F(K)=U_{\alpha} f_{\alpha}(K)$ is compact in $X$.

Let $\mathcal{M}$ denote the continuous linear functionals on $\left(C_{b}(X), \beta\right)$ and let $\mathscr{B}$ denote the continuous linear operators from $\left(C_{b}(X), \beta\right)$ into $\left(C_{b}(X), \beta\right)$. Let $G$ be a collection of continuous maps from $X$ into $X$ and for $x_{0} \in X$, let $G\left(x_{0}\right)=\left\{g\left(x_{0}\right): g \in G\right\}$.

Definition. The commutant in $\mathscr{B}$ of the operators $\left\{U_{g}: g \in G\right\}$ is $\left\{T \in \mathscr{B}: T U_{g}=U_{g} T, \forall g \in G\right\}$. Denote this commutant by $\operatorname{Comm}\left(U_{G}\right)$. The following Theorem generalizes results in [4] and [5].

THEOREM 1. Let $G$ be a semi-group with identity of continuous maps of $X$ into $X$. Assume that $\exists x_{0} \in X$ such that $G\left(x_{0}\right)=X$ and specify one map $\phi_{x}$ with $\phi_{x}\left(x_{0}\right)=x$. Assume that the map $\Phi: x \rightarrow \phi_{x}$ is continuous from $X$ into $(C(X \rightarrow X)$, c.o. $)$. Let

$$
\begin{aligned}
\mathcal{N}=\left\{L \in M: L\left(U_{\phi} f\right)=L\left(U_{\psi} f\right), \forall \phi, \psi \in G \ni \phi\left(x_{0}\right)\right. & \\
= & \left.=\psi\left(x_{0}\right), \forall f \in C_{b}(X)\right\} .
\end{aligned}
$$

Then there exists a one-to-one norm preserving correspondence between $\operatorname{Comm}\left(U_{G}\right)$ and $\mathcal{N}$ given by

$$
T f(x)=L\left(U_{\phi_{x}} f\right) \text {, for some } \phi_{x} \in G \text { with } \phi_{x}\left(x_{0}\right)=x \text {. }
$$

Proof. Given $L \in \mathcal{N}$, define $T$ by $T f(x)=L\left(U_{\phi_{x}} f\right)$. Certainly $T$ is well defined since if $\phi\left(x_{0}\right)=\psi\left(x_{0}\right)$, then $L\left(U_{\phi} f\right)=L\left(U_{\psi} f\right)$ because $L$ is in $\mathcal{N}$. The function $T f(x)$ is continuous since the maps $x \rightarrow U_{\Phi(x)} f$ $\rightarrow L\left(U_{\Phi(x)} f\right)=T f(x)$ are continuous as maps $X \rightarrow\left(C_{b}(X), \beta\right) \rightarrow$ the complex numbers. Also $T f$ is bounded and $\|T\| \leqq\|L\|$ since $|T f(x)| \leqq$ $\|L\|\left\|U_{\Phi(x)} f\right\|=\|L\|\|f\|$. Now let $\phi \in G$. Then $T$ commutes with $U_{\phi}$ since

$$
\left(T U_{\phi}\right) f(x)=T\left(U_{\phi} f\right)(x)=L\left(U_{\Phi(x)}\left(U_{\phi} f\right)\right)
$$

and, letting $\left(\phi \circ \phi_{x}\right)(y)=\phi\left(\phi_{x}(y)\right)$, we have

$$
\begin{aligned}
\left(U_{\phi} T\right) f(x) & \left.=\operatorname{Tf}(\phi(x))=\operatorname{Tf}\left(\phi\left(\phi_{x}\left(x_{0}\right)\right)\right)\right) \\
& =L\left(U_{\phi \circ \Phi(x)} f\right)=L\left(U_{\Phi(x)} U_{\phi} f\right) .
\end{aligned}
$$

To show that $T$ is in $\mathscr{B}$ it suffices to prove that $T$ is $\beta$ continuous on $\beta$ bounded sets [7]. Let $S$ be a bounded set of functions in $C_{b}(X)$. Let $\epsilon>0$ and assume $K$ is a compact set in $X$. Choose $K_{1}$ and $\delta$ such that $\|g\|_{K_{1}}<\delta$ implies $|L(g)|<\epsilon$. Let $K_{2}=\left\{\phi_{x}(y): x \in K, y \in K_{1}\right\}$. Then the 
map $\Phi: x \rightarrow \phi_{x}$ being continuous implies $\left\{\phi_{x}: x \in K\right\}$ is compact in $C(X \rightarrow X$, c.o. $)$. Hence by Lemma $3, K_{2}$ is compact.

Therefore if $\|f\|_{K_{2}}<\delta$, then we have

$$
\left\|U_{\Phi(x)} f\right\|_{K_{1}}=\sup _{g \in K_{1}}\left|f\left(\phi_{k}(y)\right)\right|<\delta, \forall x \in K
$$

and

$$
\|T f\|_{K}=\sup _{x \in K}|T f(x)|=\sup \left\{\left|L\left(U_{\Phi(x)} f\right)\right|: x \in K, \phi\left(x_{0}\right)=x\right\}<\epsilon .
$$

Now assume $T \in \operatorname{Comm}\left(U_{G}\right)$ and define $L$ by

$$
L f=T f\left(x_{0}\right) .
$$

To see that $L$ is in $\mathcal{M}$, let $S$ be a bounded set in $\left(C_{b}(X), \beta\right)$. Let $K$ be compact in $X, x_{0} \in K$, and $\epsilon>0$. Choose $K_{1}$ compact in $\left(C_{b}(X), \beta\right)$ and $\delta(\epsilon)>0$ such that if $\|f\|_{K_{1}}<\delta$ and $f \in S$, then $\|T(f)\|_{K}<\epsilon$. Then for $f$ in $S$ and $\|f\|_{K_{1}}<\delta$, we have $|L(f)|=\left|T f\left(x_{0}\right)\right| \leqq\|T f\|_{K}<\epsilon$. Thus $L$ is in $\mathcal{M}$. Now assume that $\phi$ and $\psi$ are in $G$ with $\phi\left(x_{0}\right)=\psi\left(x_{0}\right)$. Let $f$ be in $C_{b}(X)$. Then

$$
L\left(U_{\phi} f\right)=T\left(U_{\phi} f\right)\left(x_{0}\right)=U_{\phi} T f\left(x_{0}\right)=T f\left(\phi\left(x_{0}\right)\right)
$$

and similarly $L\left(U_{\phi} f\right)=T f\left(\psi\left(x_{0}\right)\right)$. Thus $L\left(U_{\phi} f\right)=L\left(U_{\psi} f\right)$ and $L \in \mathcal{N}$.

To show that the maps are inverse let $L$ be in $\mathcal{N}$ and apply (1) to obtain $T \in \operatorname{Comm}\left(U_{G}\right)$ and then apply (2) to obtain $L^{\prime} \in \mathcal{N}$. Then

$$
L^{\prime}(f)=T f\left(x_{0}\right)=L\left(U_{\Phi\left(x_{0}\right)} f\right)=L f .
$$

Now given $T \in \operatorname{Comm}\left(U_{G}\right)$ apply (2) to obtain $L \in \mathcal{N}$ and apply (1) to $L$ to obtain $T^{\prime} \in \operatorname{Comm}\left(U_{G}\right)$. Then

$$
T^{\prime} f(x)=L\left(U_{\Phi(x)} f\right)=T\left(U_{\Phi(x)} f\right)\left(x_{0}\right)=U_{\Phi(x)} T f\left(x_{0}\right)=T f(x) .
$$

Thus the maps (1) and (2) are inverse. Notice also that under (1) we have $\|T\| \leqq\|L\|$ and under (2) we have $\|L\| \leqq\|T\|$.

We obtain as a corollary the following result in [5] where $X$ had a group structure which was not necessarily abelian. For ease of exposition we assume $X$ is abelian. Then $X$ has a representation as linear operators (translation operators) on $C_{b}(X)$. For $a$ in $X$, and $f$ in $C_{b}(X)$ we define

$$
U_{a} f(x)=f(a+x) .
$$


Let $G=\left\{\phi_{a}: a \in X\right\}$ where $\phi_{a}(x)=x+a$. Notice that $\phi_{a}(0)=a$ and we are thus associating the map $\phi_{a}$ with $a \in X$ as in Theorem 1.

Corollary 1 [4]. Let $\mathscr{B}$ denote the continuous linear operators from $\left(C_{b}(X), \beta\right)$ into itself where $X$ is a locally compact Hausdorff abelian group.

Then there exists a one-to-one norm preserving correspondence between $\operatorname{Comm}\left(U_{G}\right)$ and $M$ given by

$$
T f(x)=L\left(U_{x} f\right), \quad x \in X, \quad f \in C_{b}(X) .
$$

Proof. We apply Theorem 1 with $x_{0}=0$ the identity in $X$. Then $G(0)=X$ and $\mathcal{N}=\mathcal{M}$. It only remains to be verified that the map $X \times X$ into $X$ given by $(x, y)$ into $\phi_{x}(y)$ is continuous. But if $\left\{\left(x_{\alpha}, y_{\alpha}\right): \alpha \in A\right\}$ is a net in $X \times X$ converging to $(x, y)$, then since $\phi$ is continuous $\left\{\phi_{x_{\alpha}}\left(y_{\alpha}\right)=x_{\alpha}+y_{\alpha}: \alpha \in A\right\}$ converges to $\phi_{x}(y)=x+y$.

We now apply Theorem 1 to the case when $G$ consists of analytic maps. A uniqueness set for $b$ is a subset $S$ of $D$ such that if $f, g \in B$ and $f=g$ on $S$, then $f=g$ on $D$. In order to apply Theorem 1 it is sufficient if $G\left(z_{0}\right)$ determines the functions in $B$ uniquely. Let $B^{*}$ denote. the continuous linear functionals on $(B, \beta)$.

COROllary 2. Let $G$ be a collection of analytic maps of $D$ into $D$. Assume that $G\left(z_{0}\right)$ is a uniqueness set for $B$.

Then there exists a one-to-one correspondence between $\operatorname{Comm}\left(U_{G}\right)$ and a subset of $B^{*}$.

Proof. Given $T$ in $\operatorname{Comm}\left(U_{G}\right)$, define $L$ by $L f=T f\left(z_{0}\right)$. Then $L$ is well defined and $L$ is in $B^{*}$. Indeed, if the sequence $\left\{f_{n}\right\}$ in $B$ converges $\beta$ to zero, then $\left\{T f_{n}\right\}$ converges $\beta$ to zero and so $\left\{T f_{n}\left(z_{0}\right)\right\}$ converges to zero. It suffices to consider sequences since a subset of $B$ is $\beta$ closed if and only if it is $\beta$ sequentially closed ([1], [8]).

If $T_{1}$ and $T_{2}$ are in $\operatorname{Comm}\left(U_{G}\right)$ and they both map to $L$, then for any $f$ in $B, T_{1} f\left(z_{0}\right)=T_{2} f\left(z_{0}\right)$. For any $\phi_{x}$ in $G$ with $\phi_{x}\left(z_{0}\right)=x$ we have, letting $g=\phi_{x}$,

$$
L\left(U_{g} f\right)=T_{1}\left(U_{g} f\right)\left(z_{0}\right)=U_{g} T_{1} f\left(z_{0}\right)=T_{1} f(x)
$$

and

$$
L\left(U_{g} f\right)=T_{2}\left(U_{g} f\right)\left(z_{0}\right)=U_{g} T_{2} f\left(z_{0}\right)=T_{2} f(x) .
$$

Thus $T_{1} f(x)=T_{2} f(x)$ for all $x$ in $G\left(z_{0}\right)$. Thus $T_{1} f=T_{2} f$ and $T_{1}=T_{2}$.

One can recover $T$ from $L$ by defining for $x \in G\left(z_{0}\right)$ 


$$
T f(x)=L\left(U_{g} f\right)
$$

where $g=\phi_{x}$ and $\phi_{x}\left(z_{0}\right)=x$.

Corollary 3. Let $G$ be a collection of holomorphic maps of $D$ into $D$ such that $G\left(z_{0}\right)$ is a uniqueness set for $B$. Denote by $\phi_{x}$ a distinguished element of $G$ mapping $z_{0}$ to $x, x \in G\left(z_{0}\right)$. Assume that $\phi_{x}(z)$ is analytic in $x$, for each $x \in G\left(z_{0}\right)$.

Then there exists a one-to-one correspondence between Comm $\left(U_{G}\right)$ and

$$
\mathcal{N}=\left\{L \in \mathcal{M}: L\left(U_{g} f\right)=L\left(U_{h} f\right) \forall g, h \in G \ni g\left(z_{0}\right)=h\left(z_{0}\right), \forall f \in B\right\},
$$

given by

$$
T f(x)=L\left(U_{\Phi(x)} f\right), \quad f \in B .
$$

Proof. The map from $T$ to $L$ maps $\operatorname{Comm}\left(U_{G}\right)$ into $\mathcal{N}$. We have to verify that any $L$ in $\mathcal{N}$ maps into $\operatorname{Comm}\left(U_{G}\right)$ and this only requires verification that $\operatorname{Tf}(x)$ is analytic in $x$. This follows by using Morera's Theorem, the characterization of $\mathcal{M}$ as the Radon measures on $D$ and the analyticity of $\phi_{x}(z)$ in $x$.

We consider the maps $\phi_{x}$ from $D$ into $D$ given by $\phi_{x}(z)=g(x) z$ where $g$ is a fixed analytic nonconstant function in $B$. A special case is $g(x)=x$. Denote the map $z \rightarrow g(x) z$ by $\phi_{g(x)}$. Thus $\phi_{g(x)}(z)=g(x) z$. Observe that $\phi_{g(x)}(z)$ is analytic in $x$ and $G\left(z_{0}\right)=\left\{g(x) z_{0}: x \in D\right\}$ is a set of uniqueness for $B$ for any $z_{0} \neq 0$ in $D$ because $g(D)$ is an open set.

We say that a linear operator $T$ from $B$ to $B$ is a multiplier on $B$ if there exists a sequence $\left\{c_{n}\right\}_{n=0}^{\infty}$ of complex numbers such that if $f(z)=$ $\sum a_{n} z^{n} \in B$, then $\operatorname{Tf}(z)=\sum a_{n} c_{n} z^{n}$. Let $\Delta$ denote the class of all such multipliers. It is known that $\Delta \subseteq[\beta: \beta]$, and the sequences $\left\{c_{n}\right\}$ associated with operators in $\Delta$ have been characterized [1]. In particular if $\left\{c_{n}\right\}_{n=0}^{x}$ is a sequence of complex numbers such that $\lim \sup \left|c_{n}\right|^{1 / n}<1$, then an operator $T$ defined on $B$ by $T\left(\sum a_{n} z^{n}\right)=\sum a_{n} c_{n} z^{n}$ for $\sum a_{n} z^{n} \in B$ is a multiplier in $[\beta: \beta]$.

4. Commutants of multipliers. Clearly $\Delta$ is a commutative algebra. Furthermore if $T \in[\beta: \beta]$ commutes with every operator in $\Delta$, then $T \in \Delta$. In fact a stronger result (Proposition 1) holds. First recall that an eigenvalue for a linear operator is called simple if the corresponding eigenspace has dimension one. One can easily verify that if $T \in \Delta$ has associated sequence $\left\{c_{n}\right\}$, then the eigenvalues of $T$ are simple if and only if the sequence $\left\{c_{n}\right\}$ has no repeated terms. Notice 
that $\Delta$ does actually contain operators with only simple eigenvalues. For example, let $c_{n}=(1 / 2)^{n}$. Proposition 1 follows readily.

Proposition 1. If $T \in[\beta: \beta]$ commutes with an operator $V \in \Delta$ whose eigenvalues are all simple, then $T \in \Delta$.

COROllary 4. Let $g(x)$ be a fixed nonconstant analytic function mapping $D$ into $D$. Let $G=\left\{\phi_{g(x)}: x \in D\right\}$. Then $\operatorname{Comm}\left(U_{G}\right)=\Delta$.

This follows because for any such function $g(x),|g(x)|<1$ for $x \in D$ and hence $U_{\phi_{g(x)}} \in \Delta$ and has simple eigenvalues.

Proposition 2. Let $T \in[\beta: \beta]$. Then $\operatorname{Comm}(T)=\Delta$ if and only if $T \in \Delta$ and the eigenvalues of $T$ are all simple.

Definition. For $|a| \leqq 1$, define the translation operator $U_{a}$ by $U_{a} f(z)=f(a z)$.

The operator $U_{a}$ is in $\Delta$ for $|a| \leqq 1$, and it has associated sequence $\left\{a^{n}\right\}$. Thus if $a^{n} \neq 1, \forall n$, then $\operatorname{Comm}\left(U_{a}\right)=\Delta$. For example, this holds for an operator $U_{a}$ with $|a|<1$. If $|a|=1$ and $a^{m_{n}}=1$, then $\Delta$ is a proper subset of $\operatorname{Comm}\left(U_{a}\right)$, moreover:

Proposition 3. Let $T \in[\beta: \beta]$ and $a^{n_{0}}=1$. Then $T \in \operatorname{Comm}\left(U_{a}\right)$ if and only if $u_{n}^{(m)}=0$ whenever $n-m \neq n_{0} s$ for $s$ an integer, where $T\left(z^{n}\right)=u_{n}(z)$.

It is not known which operators with these sorts of gaps in the sequence $\left\{c_{n}\right\}$ are in $[\beta: \beta]$. However, there certainly are some in $[\beta: \beta]$ which are not in $\Delta$. For example, for a fixed $n_{0}$, define the operator $T$ by

$$
T\left(z^{n}\right)= \begin{cases}0 & n \neq 1 \\ z+z^{1+n_{0},} & n=1 .\end{cases}
$$

One expects in a given situation, that if $G$ is "large" enough then Comm $(G)$ will consist only of constant multiples of the identity operator I.

Proposition 4. Let $G \subseteq[\beta: \beta]$ and assume

(i) $G$ contains at least one multiplier all of whose eigenvalues are simple and

(ii) $G$ contains one linear fractional transformation $U_{\phi}$ where $\phi(z)=$ $(z-a) /(1-\bar{a} z)$, with $0<|a|<1$. 


\section{Then Comm $G=\{c I: c$ complex $\}$.}

Finally we observe that for a given operator $V \in[\beta: \beta], \operatorname{Comm}(V)$ will contain all power series in $V$ which converge to an element in $[\beta: \beta]$. But, in particular, for the operator $U_{a}$, for $|a|<1$, it can be shown that not all operators in $\operatorname{Comm}\left(U_{a}\right)$ can be obtained by power series.

\section{REFERENCES}

1. M. W. Bartelt, Multipliers and operator algebras on bounded analytic functions, Pacific J. Math., 37 (1971), 575-584.

2. - Approximation in operator algebras on bounded analytic functions, Trans. Amer. Math. Soc., 170 (1972), 71-83.

3. R. C. Buck, Operator algebras and dual spaces, Proc. Amer. Math. Soc., 3 (1952), 681-687.

4. - Algebras of linear transformations, Technical Report \#4 under OOR contract TB2-001 (1406), 1956.

5. Algebraic properties of classes of analytic functions, seminars on Analytic Functions, Vol.

II, Princeton, 1957, 175-188.

6. Bounded continuous functions on a locally compact space, Michigan Math. J., 5 (1958), $95-104$.

7. J. R. Dorroh, The localization of the strict topology via bounded sets, Proc. Amer. Math. Soc., 20 (1969), 413-414.

8. L. A. Rubel and J. V. Ryff, The bounded weak-star topology and the bounded analytic functions, J. Functional Analysis, 5 (1970), 167-183.

9. L. A. Rubel and A. L. Shields, The space of bounded analytic functions on a region, Ann. Inst. Fourier (Grenoble) 16 (1966), fasc. 1, 235-277.

10. A. L. Shields and L. J. Wallen, The commutants of certain Hilbert space operators, Indiana Univ. Math. J., 20 (1971), 777-788.

Received September 25, 1974.

Rensselaer Polytechnic Institute

AND

Christopher Newport College 


\section{PACIFIC JOURNAL OF MATHEMATICS}

\section{EDITORS}

RICHARD ARENS (Managing Editor)

University of California

Los Angeles, California 90024

\section{J. Dugundu}

Department of Mathematics University of Southern California Los Angeles, California 90007

D. Gilbarg and J. Milgram Stanford University Stanford, California 94305

\section{ASSOCIATE EDITORS}
E. F. BECKENBACH
B. H. NeumanN
F. WOLF
K. YoSHIDA

\section{SUPPORTING INSTITUTIONS}

UNIVERSITY OF BRITISH COLUMBIA CALIFORNIA INSTITUTE OF TECHNOLOGY

UNIVERSITY OF CALIFORNIA

MONTANA STATE UNIVERSITY

UNIVERSITY OF NEVADA

NEW MEXICO STATE UNIVERSITY

OREGON STATE UNIVERSITY

UNIVERSITY OF OREGON

OSAKA UNIVERSITY

\author{
UNIVERSITY OF SOUTHERN CALIFORNIA \\ STANFORD UNIVERSITY \\ UNIVERSITY OF HAWAII \\ UNIVERSITY OF TOKYO \\ UNIVERSITY OF UTAH \\ WASHINGTON STATE UNIVERSITY \\ UNIVERSITY OF WASHINGTON \\ AMERICAN MATHEMATICAL SOCIETY
}

The Supporting Institutions listed above contribute to the cost of publication of this Journal, but they are not owners or publishers and have no responsibility for its contents or policies.

Mathematical papers intended for publication in the Pacific Journal of Mathematics should be in typed form or offset-reproduced (not dittoed), double spaced with large margins. Underline Greek letters in red, German in green, and script in blue. The first paragraph or two must be capable of being used separately as a synopsis of the entire paper. Items of the bibliography should not be cited there unless absolutely necessary, in which case they must be identified by author and Journal, rather than by item number. Manuscripts, in duplicate, may be sent to any one of the four editors. Please classify according to the scheme of Math. Reviews, Index to Vol. 39. All other communications should be addressed to the managing editor, or Elaine Barth, University of California, Los Angeles, California, 90024.

100 reprints are provided free for each article, only if page charges have been substantially paid. Additional copies may be obtained at cost in multiples of 50 .

The Pacific Journal of Mathematics is issued monthly as of January 1966. Regular subscription rate: $\$ 72.00$ a year (6 Vols., 12 issues). Special rate: $\$ 36.00$ a year to individual members of supporting institutions.

Subscriptions, orders for back numbers, and changes of address should be sent to Pacific Journal of Mathematics, 103 Highland Boulevard, Berkeley, California, 94708.

PUBLISHED BY PACIFIC JOURNAL OF MATHEMATICS, A NON-PROFIT CORPORATION

Printed at Jerusalem Academic Press, POB 2390, Jerusalem, Israel.

$$
\begin{gathered}
\text { Copyright } 1976 \text { Pacific Journal of Mathematics } \\
\text { All Rights Reserved }
\end{gathered}
$$




\section{Pacific Journal of Mathematics}

Vol. 62, No. 2

February, 1976

Allan Russell Adler and Catarina Isabel Kiefe, Pseudofinite fields, procyclic

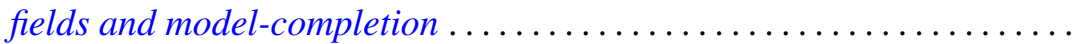

Christopher Allday, The stratification of compact connected Lie group

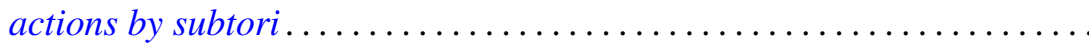

Martin Bartelt, Commutants of multipliers and translation operators .......

Herbert Stanley Bear, Jr., Ordered Gleason parts ..................

James Robert Boone, On irreducible spaces. II .....................

James Robert Boone, On the cardinality relationships between discrete

collections and open covers ............................

L. S. Dube, On finite Hankel transformation of generalized functions .......

Michael Freedman, Uniqueness theorems for taut submanifolds . . . . . . . . .

Shmuel Friedland and Raphael Loewy, Subspaces of symmetric matrices

containing matrices with a multiple first eigenvalue .............

Theodore William Gamelin, Uniform algebras spanned by Hartogs

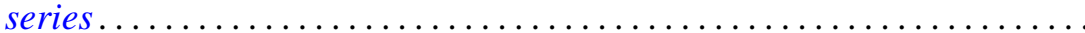

James Guyker, On partial isometries with no isometric part ............

Shigeru Hasegawa and Ryōtarō Satō, A general ratio ergodic theorem for

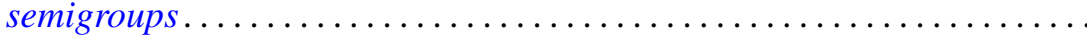

Nigel Kalton and G. V. Wood, Homomorphisms of group algebras with norm less than $\sqrt{2}$.

Thomas Laffey, On the structure of algebraic algebras...

Will Y. K. Lee, On a correctness class of the Bessel type differential operator $S_{\mu}$

Robert D. Little, Complex vector fields and divisible Chern classes ....

Kenneth Louden, Maximal quotient rings of ring extensions . .

Dieter Lutz, Scalar spectral operators, ordered $l^{\rho}$-direct sums, and the

counterexample of Kakutani-McCarthy . .

Ralph Tyrrell Rockafellar and Roger Jean-Baptiste Robert Wets, Stochastic

convex programming: singular multipliers and extended duality

singular multipliers and duality.

Edward Barry Saff and Richard Steven Varga, Geometric overconvergence of rational functions in unbounded domains ..........

Joel Linn Schiff, Isomorphisms between harmonic and P-harmonic Hardy

spaces on Riemann surfaces.

Virinda Mohan Sehgal and S. P. Singh, On a fixed point theorem of

Krasnoselskii for locally convex spaces.

Lewis Shilane, Filtered spaces admitting spectral sequence operations

Michel Smith, Generating large indecomposable continua . 